\title{
Some Strongly Almost Summable Sequence Spaces
}

\author{
Sunil K. Sharma and Ayhan Esi
}

ABSTRACT: In the present paper we introduce some strongly almost summable sequence spaces using ideal convergence and Musielak-Orlicz function $\mathcal{M}=\left(M_{k}\right)$ in $n$-normed spaces. We examine some topological properties of the resulting sequence spaces. 